\title{
Through A Cracked Lens: Alternate Views of Light Microscopy. Part I: Why Does My Microscope Have A Pupil?
}

Mark W. Lund, Ph.D, MOXTEK, Inc.

Let's face it, optics is a fascinating field, but it can bore you to death. My purpose in writing these articles is to show that there are simple, intuitive ways to look at microscopes that can illuminate rather than bore. I have been an optical engineer for many years and have observed many unique ways of looking at optical instruments. I hope that I can share my enthusiasm and insight for light and its manipulation and give some insights that microscopists may have missed. The microscope is particularly interesting because the glass lenses often do more than one job at the same time. Most of the principles of optical microscopy are directly transferable to electron microscopy, and I will point out specific cases as I go along.

This month I want to talk about something so fundamental, and yet so interesting that most microscopy texts don't even mention it. Microscopy texts are supposed to be boring! Using this concept I will comment on your probable first experience with a microscope. Figure 1 shows what you may have saw the very first time you looked into a microscope. Likely as not, the next time you looked you saw something similar to Figure 2. Even the best microscopists still see scenes like these, but automatically know what to do about it.

Now I will explain what you saw, so you can tell beginners what they are seeing and why. I have not been able to find the following information in any text.

Every single visual optical instrument has what is called an exit pupil. Wait a minute, don't glaze over yet, it's just a name! There are other names for it: eyepoint, Ramsten Circle, Larynges disc, and eye ring. The exit pupil of a microscope is easy to find. Take a thin piece of paper and hold it a few $\mathrm{mm}$ behind the eyepiece, where your eye would normally go. If the light is on, and the microscope is focused on something reasonably bright, you will be able to find a small dot of light hanging in the air. By waving the paper about a bit you will find that the light comes to a very nice focus, revealing a sharp bright disk. The larger the magnification of the objective lens, the smaller the pupil.

Get some binoculars and point them at a bright scene. You can find their exit pupils (one for each eye) exactly the same way, but they will be much larger than the microscope pupils. A rifle scope will have an even larger exit pupil, but it will be a few $\mathrm{cm}$ away from the eyepiece. If you examine the exit pupil carefully (it is easier with the binoculars or rifle scope), you will find that it does not contain a recognizable picture. It seems to be (and is) uniformly bright. In fact, by poking around with your piece of paper you will discover that all the light coming out of the eyepiece goes through the exit pupil.

Now you are familiar with another pupil - -the pupil of your eye, that organ that you see giving you a skeptical look every morning in the mirror. If you ignore its tendency to be critical and look at it closely, you find that the eye's pupil is a small black spot. Its main feature is that all the light coming into the eye must pass through it. It is actually the entrance pupil of the eye

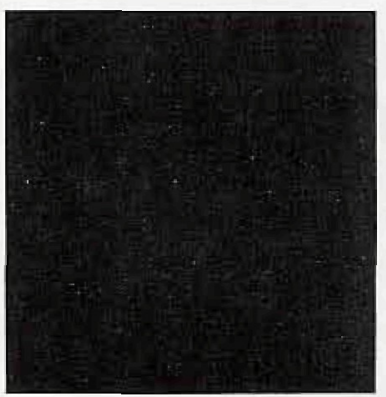

Figure 1. Artists concplion of what you likely saw the first time you looked in an uptical microscope

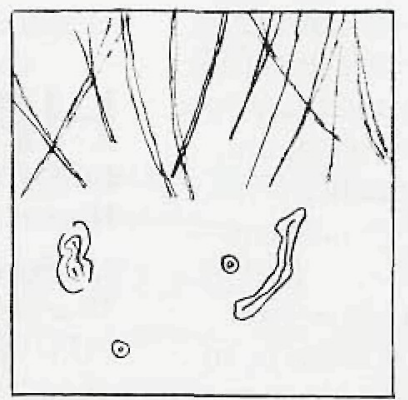

Figure 2 Artist conception of what you likely saw the next time you looked in an optical microscope
Starting to sound familiar? We are now ready to give one of the great truths of optics: if you don't put the exit pupil of the first instrument in the entrance pupil of the next, you will be very disappointed in the result. Why? Because unless you have the pupils aligned you will lose a lot of important light and won't be able to see the (full) image. Now we understand why Figure 1 is so often seen. Having a perfect eye behind the eyepiece of a great microscope isn't enough. You have to have the exit and entrance pupils lined up, which takes a bit of practice-- particularly when you have a binocular microscope.

How can you see a wide-screen-technicolor image when the exit pupil is the size of a pinhead? It is exactly like looking through a knothole. You want to see what's going on behind the fence. If you stand back from the knothole you see a very small field of view - just the third baseman's shirt. Light from every point of the baseball field is always going through the knothole, but the shaft of light coming from the pitcher is hitting you in the shoulder, not in the eye. As you get closer you can see farther to the sides because the shafts of light are converging on the knothole. When you get your entrance pupil into the knothole the fence is no longer restricting your view of the game

In this same way the microscope exit pupil consists of narrow pencils of light about the same diameter as the exit pupil, but going in different directions. If your eye's pupil is behind the exit pupil, the field of view is restricted because some of the pencils are striking your forehead and nose, not getting into your eye. You have seen this effect many times with binoculars, telescopes, microscopes, etc. You get a blackout or narrow view until your eye is in the right place. You get the full field of view when the exit pupil of the microscope is in the entrance pupil of your eye.

In Figure 2, you saw something very strange. You hadn't focused the microscope yet, but you were seeing weird stuff that moved when you blinked and that moved around quickly when you looked in different directions. Once you caught on that you were looking at eyelashes and floaters you wondered "How can they be magnified so much?" To explain Figure 2, I need to explain what the exit pupil really is. Now this is very easy: The exit pupil is the image that the eyepiece makes of the objective lens. In other words, the eyepiece is a lens and the brightest thing that it sees is the objective, some $160 \mathrm{~mm}$ away.

Is it confusing to think about an image of a lens, instead of by a lens? Well, if there were a lens sitting on a table you could see it, so your eye is making an image of the lens. If you held the lens up to the sky the lens would be full of light. and so you would see it as a bright lens. In this sense the eyepiece doesn't care about what else the objective is doing to the image. It just sees it as a bright object $160 \mathrm{~mm}$ away and makes an image of it. Now this is interesting because it explains why this image is called the exit pupil. Any light coming through the microscope has to go through the objective lens, and so it also has to go through the image of the objective lens (i.e., the exit pupil)

The exit pupil of a microscope is very small. The objective lens is small to begin with, and the image is demagnified by a factor of five (assuming a $10 \mathrm{x}$ eyepiece). An inexpensive microscope with an $80 x$ objective like the one you used in junior high school will have an exit pupil of less than 200 microns (expensive ones you use today can be as large as 600 microns). This means that all the light pencils that intersect at the exit pupil are about 200 microns in diameter. An eyelash in the way of these pencils will cast a giant shadow on your retina. Likewise, any dust on your cornea and 'floaters' inside your eye will suddenly appear as highly magnified shadows. You can see these because the pupil of your eye is not filled. In normal vision the shadow cast by an eyelash is overwhelmed because the blocked light is only a small fraction of the total entering the pupil. Each pencil from each point in your field of view will completely fill your eye's entrance pupil. Shadows cast by eyelashes and floaters are overwhelmed by other light going around them. This effect is worse with high power objectives because their exit pupils are smaller--more light is blocked by an eyelash, and sharper shadows result.

Now for you advanced optical and electron microscope enthusiasts: As many of you have already guessed, the pupils are images of the aperture stops. Next month I will discuss field stops and aperture stops and the microscope's two separate but merged imaging systems-one which carries the image, and the other which carries the light

I hope readers will give me some idea of light microscopy topics of interest you. If I am not able to respond, I will find someone that can. Contact me at MOXTEK, at lundm@x-ray byu edu or in care of Microscopy Today 


\section{FLOW CYTOMETRY}

M.G. Ormerod, Consultant, Surrey, U.K

Flow cylometry is a specialized form of microscopy for measuring the properties of single cells. Flow cytometers are becoming widely used in clinical and research laboralores and there is an increasing need for non-specialists to have an understanding of this technology. Flow Cytometry is a practical guide which covers both routine applicaions of flow cytometry and the more recent applications of this exciting technology.

Contents: What is flow cytometry, Instrumentation, Fluorescence Immunofluorescence Analysis of DNA. Study of cell proliferation and death, Other applications. Appendices Glossary Suppliers, Learned societies.

Readership: Postgraduates, clinicians, researchers, and all first-time users. Paperback 88 pp; 1994

$\$ 27.00$ plus $\$ \& H^{*}$

\section{MODERN PHOTOMICROGRAPHY}

B. Bracegirde \& S. Bradbury

A completely new practical guide to photomicrography and a valuable companion viume to Scientific PhotoMACROgraphy by Brian Bracegirdle. Illustration of results is such an mportant part of communication in microscopy and this book is packed with useful advice on choosing equipment, correct lechnique and obtaining first class results

Contents (provisional): Obtaining the image Recording the image in monochrome and in colour Special problems and lechniques. Publishing the results Suppiters

Readership: All who wish to record photographic images, whether novices or experienced workers.

Paoerbach 0112 p

$\$ 33.00$ plus $S \& H^{*}$

\section{SCIENTIFIC PHOTOMACROGRAPHY}

B Bracegirdle, Cheltenham, UK

Although modern equipment makes it easier to obtain high quality photographs of scientific specimens at low magnification, the choice and use of the most approprate procedure is still critical

Scientific PhotoMACROgraphy is a detaled practical guide to choosing the correct equipment and methods for boih transmitted-light and reflected-ight photography Extensive reference dala are provided in readily-accessible nomograms and tables and some 40 half-tones show how the equipment should be set up

Bv following the advice given here, the reader should be able to produce high quality photographs on a routine basis.

Contents: The scope of the process, Obtaining the magnification Working with transmited light; Working with reflecied light, General remarks on illumination and exposure Estumating exposure in macro-range photography. Recording the image

Readersinip: 2nd year undergraduates and above, any researcher using the microscope at low resolution

Paperback 120 pp, January 1995 S31.00 pus S\&H*

\section{MICROSCOPY OF TEXTILE FIBRES}

\section{$\mathrm{PH}$ Greaves \& BP Savile}

Recent advances in microscopy, logether with developments in fibre technology and changing commercial prionities have created a great need for this up-io-date manual

The book is a practical guide to the properties and characteristics of textile fibres with clear advice on sampling. specimen preparation and examination procedures. Writ= ten with todays technological and commercial requirements in mind, the subjects covered include fibre identification, scanning and transmission electroscopy, and speciality fibre analysis. The potentials of the new confocal and infra-red microscopes are also introduced

Contents: Intraduction. Fibre identification, Fibre measurement Polarised ligh microscopy, Special preparation techniques for light microscopy, Other light microscoplcal techniques applied to fibres; Scanning electron microscopy. Transmission electron microscopy

Readership: Texhle technologists, Fibre scientists; Microscopists, Forensic scientisis Materials analysis

Paperback 112 pp, March 1995

$\$ 3300$ plus $S \& H^{x}$

\section{FOOD MICROSCOPY}

O. Flint, University of Leeds, Leeds, UK

Food Microscopy is a practical guide to examining the microstructure of food ingredients and food products using the light microscope. The book covers a range of practical lechniques with the emphasis on rapid methods which have been tried, tested and modified by the author to ensure that they work.

After reading this book a microscopist should be able to select and modify the techniques to make them suitable for individual food products.

Contents: Introduction, Choice of equipment, Preparation of food for the stereomicroscope; Simple preparation techniques for the compound microscope; Use of the cryostat in food microscopy, Contrast techniques for food constituents, Fat in Food, Food starches: Meat, fish and their products; Vegetable proteins; The Howard mould count of tomato products; Food gums, Food emulsions.

Readership: Undergraduates, postgraduates and researchers in food science, industrial food scientists

Paperback, 144 pp, 1994

$\$ 31.00$ plus $\$ \& H^{*}$

\section{BIOLOGICAL MICROTECHNIQUE}

J. Sanderson, Sir William Dunn School of Pathology, Oxford, UK

Describing both new and classical methods of slide-making, this book contains a wealth of practical detall to provide a firm grounding in preparative methods for light microscopy. It will appeal to both novices and experienced microscopists seeking to extend their repertore of lechniques.

Contents: Fixation, Tissue processing, Microtomy, Other preparalive methods Staining and dyeing; Finishing the preparation. Appendices. Safety, Refractive indices

Readership: Researchers, technicians, amateur microscopists, undergraduates school science teachers and students.

Paperback 240 pp. 1994

$\$ 39.00$ plus S\&H*

\section{ADDITIONAL RMS MICROSCOPY HANDBOOKS}

01 An Introduction to the Optical Microscope $\$ 2700$ plus $\$ \& \mathrm{H}^{*}$

03 Specimen Preparation for Transmission Eleciron Microscopy of Materials $\$ 2100$ plus $S \& H^{*}$

04 Histochemical Protein Staining Methods. \$21.00 plus S\&H*

06 Liquid Histochemistry. $\$ 21.00$ plus $\mathrm{S \& H}$

08 Maintaining and Moniloring the Transmission Electron Microscope: $\$ 21.00$ plus S\&H

09 Qualitative Polarized-Light Microscopy. $\$ 31.00$ plus $\mathrm{S} \& \mathrm{H}^{*}$

11 An Introduction to Immunocytochemistry. $\$ 21.00$ plus $\$ \& H^{*}$

15 RMS Dictionary of Light Microscopy: $\$ 41.00$ plus S\&H*

16 Light-element Analysis in the TEM WEDX and EELS $\$ 21.00$ plus S\&H*

17 Colloidal Gold $\$ 2100$ plus S\&H*

18 Autoradiography A Comprehensive Overview: $\$ 27.00$ plus ${\mathrm{S} \& \mathrm{H}^{*}}^{*}$

19 Introduction to Crystallography $\$ 27.00$ plus $S \& H^{*}$

21 Cryopreparation of Thin Biological Specimens for Electron Microscopy $\$ 2700$ plus $S \& H^{*}$

22 An Introduction to Surface Analysis by Electron Spectroscopy: $\$ 2700$ plus $\mathrm{S} \& \mathrm{H}^{*}$

23 Basic Measurement Techniques for Light Microscopy. $\$ 2700$ plus $\mathrm{S} \& \mathrm{H}^{*}$

24 The Preparation of Thin Sections of Rocks, Minerals, and Ceramics. S31 00 plus $S \& H^{*}$

25 The Role of Microscopy in Semiconductor Falure Analysis $\$ 31.00$ plus $\mathrm{S} \& \mathrm{H}^{*}$

26 Enzyme Histochemistry: $\$ 31.00$ plus $S \& H^{*}$

*Shipping \& Handling (U.S.): \$5 first book, $\$ 3$ each thereafter From from Microscopy Today by Visa/MasterCard, check or company P.O Microscopy Today

PO Box 122

Tel.: (608)836-1970

Middleton, WI 53562
Fax: (608)836-1969

eMail: MicroToday@aol.com 


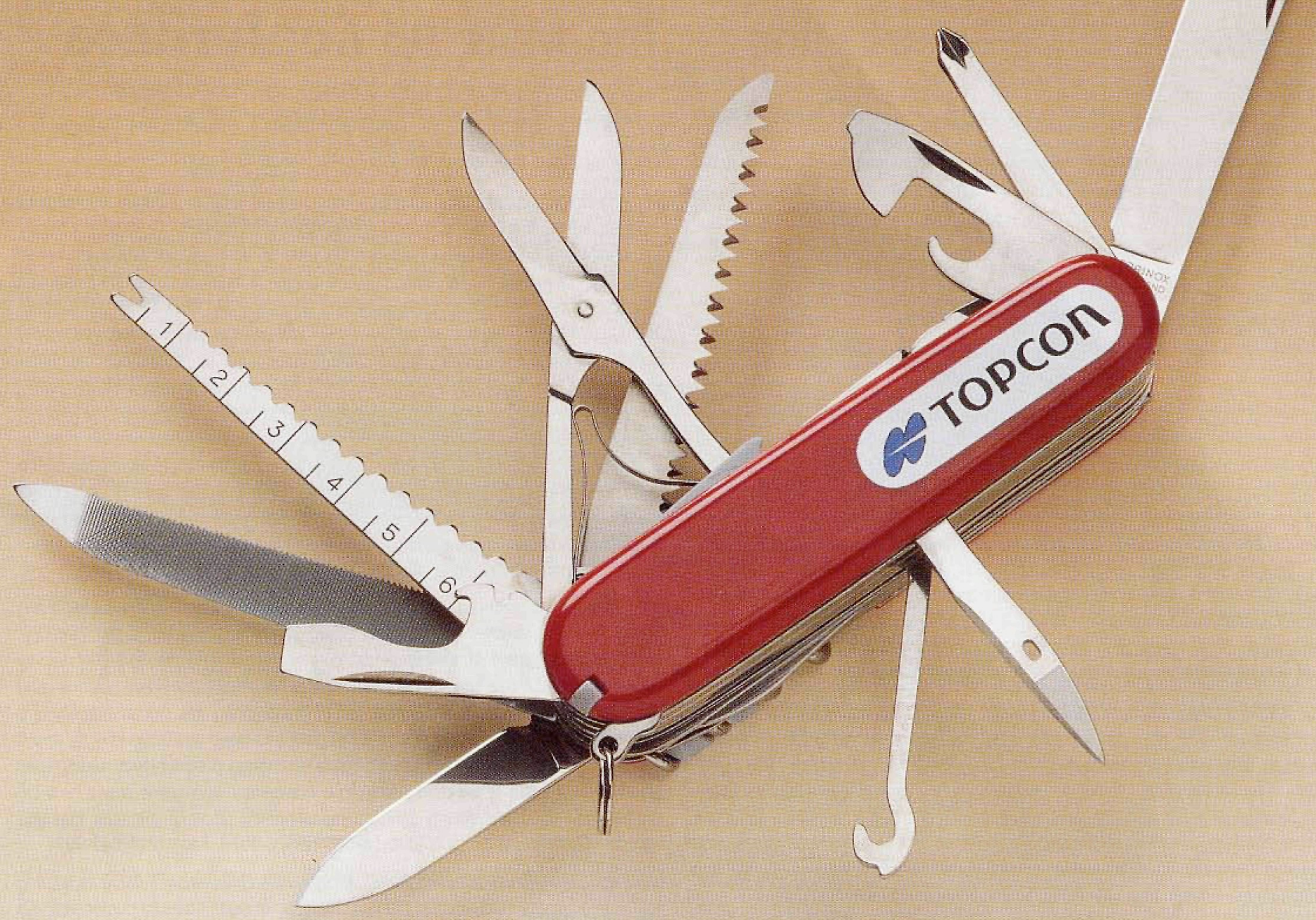

\section{UNEQUALED VERSATILITY}

\section{Topeon Scanning Electron Mieroscopes in a Unique Class}

You value the versatility of a Swiss Army Knife. Now, you can have that same kind of versatility with Topcon's 500 Series SEMs for your scanning electron microscopy tasks. The versatility to meet your needs today and tomorrow.

\section{Ultimate SEM Capabilities}

Choose from a complete family of SEMs Tungsten (W), $\mathrm{LaB}_{6}$, and Field Emission.

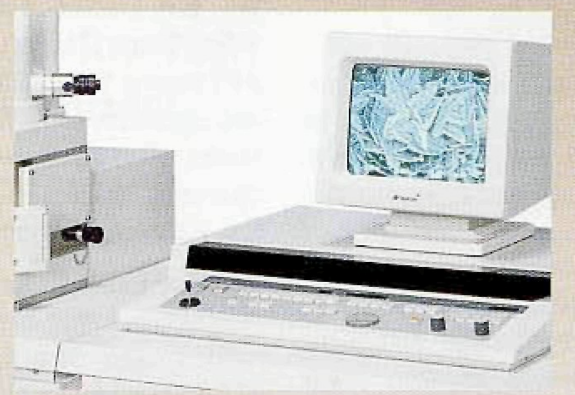

All have windows mouse-based computer controls or traditional automated controls, or both, to provide access to all SEM func- tions. Comprehensive digital imaging and archiving provide full image manipulation. Unique WET-SEM capability gives you the real image quickly and easily.

Compare and you'll agree that no other SEM comes close in versatility, ease of use, reliability and value. It's unequaled. But see for yourself. Call us at $1-800-538-6850$ or write to TOPCON, 37 West Century Road, Paramus, New Jersey 07652.

\section{TOPCON}

TOPCON TECHNOLOGIES INCORPORATED 